\title{
Research Paper: Determining Undeclared Synthetic Pharmaceuticals as Adulterants in Weight Loss Herbal Medicines
}

Babak Salahshour ${ }^{1,2}$, Sajjad Sadeghi ${ }^{1,2}$, Hajar Nazari $^{3}$, Kambiz Soltaninejad $^{2 *}$ (D)

1. Legal Medicine Center of North Khorasan Province, Bojnurd, Iran.

2. Department of Forensic Toxicology, Legal Medicine Research Center, Legal Medicine Organization, Tehran, Iran.

3. Department of Epidemiology, School of Public Health, Iran University of Medical Sciences, Tehran, Iran.

\begin{tabular}{l|l}
$\begin{array}{l}\text { Use your device to scan } \\
\text { and read the article online }\end{array}$ \\
ants in Weight Loss Herbal Medicines. International Journal of Medical Toxicology and Forensic Medicine. 2020; 10(1):26253. \\
https://doi.org/10.32598/ijmtfm.v10i1.26253
\end{tabular}

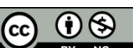

Article info:

Received: 04 Jul 2019

First Revision: 07 Jul 2019

Accepted: 14 Jul 2019

Published: 03 Apr 2020

\section{Keywords:}

Adulteration, Caffeine, Trimethoxyamphetamine

\section{ABSTRACT}

Background: The popularity and use of herbal medicines and supplements are growing worldwide. Herbal anti-obesity products have been considered as suitable alternatives to synthetic pharmaceuticals as they are introduced as harmless natural products. However, some manufacturers often add undeclared synthetic pharmaceuticals to the anti-obesity herbal medicine products to improve their efficacy and potency. The present study aimed to analyze herbal weight loss products collected from the drug market in Bojnurd City, Iran.

Methods: Ninety-six herbal drug samples, as weight loss products, were obtained from herb shops and pharmacies in Bojnurd City, Iran. All samples were analyzed to detect undeclared active pharmaceutical ingredients using High-Performance Liquid Chromatography with Diode Array Detector (HPLC-DAD) and Gas Chromatography-Mass Spectrometry (GC-MS) techniques.

Results: Caffeine, trimethoxyamphetamine, and vitamin E were identified in herbal weight loss products. Caffeine was detected in $21.8 \%$ of the obtained samples, as the most common undeclared active pharmaceutical adulterant.

Conclusion: Undeclared active pharmaceutical ingredients in herbal weight loss products could threaten patients' health. Thus, it is necessary to create awareness through health authorities in this regard.

\section{Introduction}

$\mathbf{T}$

he popularity and use of herbal medicines and supplements are increasing globally, i.e., because of numerous adverse effects of syn- thetic pharmaceuticals $[1,2]$. Herbal remedies should be considered as safe, harmless, and without adverse effects. They are used for many conditions, such as substance use disorder treatment, sexual performanceenhancing, endocrine disorders, and obesity treatment

* Corresponding Author:

Kambiz, Soltaninejad, PhD.

Address: Department of Forensic Toxicology, Legal Medicine Research Center, Legal Medicine Organization, Behesht Street, Tehran, Iran Tel: +98 (21) 55613731

E-mail: kamsoltaninejad@gmail.com 
$[3,4]$. The therapeutic modalities with herbal remedies have been effective in treating some disorders; however, many of their therapeutic dimensions remain doubtful, and their use is poorly established. Inadequate data about the mode of action, potential adverse effects, adverse reactions, toxicity, contraindications, and drug-drug or drug-food interactions are the key limitations in the safety and rational use of herbal medicine products [2].

Additionally, the adulteration of herbal medicinal products with undeclared illegal or synthetic drugs is associated with some serious health problems. Therefore, detecting these adulterants is a global concern about the purity and safety of commercially phytomedicines [5]. The most severe adverse effects caused by these adulterations include hepatic encephalopathy, hepatorenal syndrome, liver injury, cardiotoxicity, rhabdomyolysis, nephrotoxicity, metabolic acidosis, renal failure, cerebral hemorrhage and edema, coma, and death [6-8].

Globally, the prevalence of obesity has been considered as a major risk for public health. Obesity is associated with numerous pathophysiological conditions, such as cardiovascular diseases, endocrine and metabolic disorders, osteoarthritis, inflammatory rheumatic disease, and cancers [9-11]. Accordingly, the demand for antiobesity supplements has increased worldwide. Herbal anti-obesity products have been considered as suitable alternatives to synthetic pharmaceuticals, as they are introduced as harmless natural products [12].

However, some manufacturers often add undeclared synthetic pharmaceutical drugs to anti-obesity herbal medicine products to improve their efficacy and potency [12]. Previous studies in many countries, including Iran, have identified the most common undeclared active pharmaceutical ingredients as adulterants in weight loss herbal supplements from many pharmacological categories. These classes include amphetamine analogs, as anorexic agents (sibutramine, desmethyl sibutramine, amfepramone, fenproporex, fenfluramine, ephedrine, norephedrine, phendimetrazine), methylxanthines (caffeine), thyroid hormones, rimonabant, laxatives (phenolphthalein, docusate sodium), diuretics (furosemide, bumetanide, spironolactone, and hydrochlorothiazide), antidepressants (fluoxetine, paroxetine, sertraline, protriptyline, and bupropion), synthetic Phosphodiesterase type-5 (PDE5)- inhibitory drugs (sildenafil, vardenafil, and tadalafil), and opioids (methadone, tramadol) [1, 12-19].

However, a long consumption of adulterated weight loss herbal products may cause severe adverse effects and toxicity to patients and could be considered as a cause of morbidity and mortality [12, 14-18]. Therefore, the chemical analysis of adulterated herbal products is an essential issue in clinical and forensic medicine and toxicology [19]. Studies on investigating adulterants in weight loss herbal products are limited in Iran [12, 16, 19]. Thus, this study aimed to identify the possible adulteration in herbal weight loss products available in the drug market of Bojnurd City, Iran.

\section{Materials and Methods}

Methanol, ethanol, chloroform, acetonitrile, potassium dihydrogen phosphate, phosphoric acid, hydrochloric acid (37\%) were obtained (Merck Co.; Darmstadt, Germany). High-Performance Liquid Chromatography (HPLC) grade water was purchased (Merck Millipore Co.). All the used chemicals and solvents were of analytical grade.

Initially, the pharmacies and herbal shops in Bojnurd City were identified. Then, they were divided according to geographical regions, population density, and the location of herbal shops and pharmacies. All brands of herbal products sold as weight loss and slimming supplements have been randomly purchased (3 samples were randomly selected from each product's brand). Finally, 96 hand-made and factory-made samples were gathered in different pharmaceutical dosage forms (tablets, capsules, powders, topical gels, and oral drops) from herb shops over the one year study period (2018) (3 samples from 32 formulations). All of the collected samples had labels indicating herbal supplements and natural products and were advised by sellers for weight loss proposes. All study samples were introduced to the Laboratory of Forensic Toxicology, Legal Medicine Center (Bojnurd, Iran) for the analysis of synthetic pharmaceutical adulterants.

For each solid pharmaceutical dose form, organoleptic characteristics, including the features of the sample, weight, size, shape, odor, and color, were recorded. Furthermore, for liquid samples, $\mathrm{pH}$ was determined by the $\mathrm{pH}$ meter as other physicochemical characteristics (827 $\mathrm{pH}$ lab, Metrohm, Switzerland).

For powders, the sample was homogenized and uniformed with mortar and pestle. Tablets were crushed and homogenized with mortar and pestle. For capsules, after breaking the capsules, the solid content was homogenized. All obtained samples were ground to fine and uniform powders, then extracted with $3 \mathrm{~mL}$ methanol (for each1 $\mathrm{mg}$ of the sample) for $20 \mathrm{~min}$ in a test tube, using a rotator. The extract was centrifuged ( 5 minutes at 4000 rpm). The supernatant was collected and the top layer 
of the compound was injected to analytical instruments for analyses [12]. For liquid samples, the sample was diluted with methanol and filtered by a $0.22 \mu \mathrm{m}$ membrane filter (Macherey-Nagel, Germany), then injected to the instruments.

All of the samples were analyzed with the previouslyvalidated High-Performance Liquid Chromatography (HPLC) and Gas Chromatography-Mass Spectrometry (GC-MS) techniques for systematic toxicological analysis [19]. The HPLC analysis was isocratically conducted on a C18 column $(250 \mathrm{~mm} \times 4.0 \mathrm{~mm}$ ID., $5 \mu \mathrm{m}$, Eurospher II $\left.{ }^{\circledR} 100-5\right)$ using a C18 guard column (Knauer, Berlin, Germany). Analyses were performed using an HPLC system (AZURA, Knauer, Berlin, Germany) with a quaternary pump and equipped with a Diode Array Detector (DAD, S2800), and a Smart 1000 pump. A mixture of acetonitrile and phosphate buffer $(\mathrm{pH}=2.3)$ $(63: 37 \mathrm{v} / \mathrm{v})$ was used as elution solvents. A $20-\mu \mathrm{L}$ sample was injected into the column and eluted at room temperature with a constant flow rate of $1.0 \mathrm{~mL} / \mathrm{min}$.

A GC-MS (7890/5977 model, Agilent, USA) equipped with a split/splitless injector was applied. The GC column was HP-5 MS (5\% phenyl, and 95\% dimethyl polysiloxane, $30 \mathrm{~m}$ length, $0.25 \mathrm{~mm}$ ID, $0.25 \mu \mathrm{m}$ film thickness) (Agilent, USA). Mass analyzer (Quadrupole, Agilent, USA) was connected to the column. The injection port temperature was $280^{\circ} \mathrm{C}$; the transfer line temperature was $310^{\circ} \mathrm{C}$. The initial column oven was set to $60^{\circ} \mathrm{C}$ and held constant for 1 minute. Temperature program rate was $10^{\circ} \mathrm{C} / \mathrm{min}$ and final temperature was set to $310^{\circ} \mathrm{C}$ and final hold for 10 minutes. Helium (99.99\% purity) was implemented as a carrier gas with a constant flow rate of $1 \mathrm{ml} / \mathrm{min}$. The mass spectrometer was operated by electron impact $(70 \mathrm{eV})$ in positive full scan mode $(50-550 \mathrm{~m} / \mathrm{z})$. Wiley, National Institute of Standards and Technology (NIST), and Maurer/ Pfleger/ Weber (MPW) (MPW; 2011) libraries were used for the qualitative analysis of samples.

Statistical analysis was performed in SPSS. The obtained results are presented as frequency and percentages.

\section{Results}

We investigated the organoleptic characteristics of the study samples. The samples obtained as weight loss or slimming herbal products in the study were in capsules $(37.5 \%)$, water distilled $(25 \%)$, tablets $(12.5 \%)$, herbal tea $(9.37 \%)$, powders $(6.25 \%)$, oral drop $(6.25 \%)$, and gel $(3.13 \%)$ formulations. Table 1 summarizes the brand name, organoleptic characteristics, and detected undeclared pharmaceutical ingredients in the studied samples.

The qualitative analysis of the study samples indicated that $47 \%$ of all pharmaceutical dose forms contained caffeine as an active pharmaceutical ingredient. However, only $21.8 \%$ of the obtained samples were adulterated by undeclared active pharmaceuticals. Caffeine was detected in $21.8 \%$ of the study samples as the most common undeclared active pharmaceutical adulterants (Table 1), (Figure 1). Vitamin E and Trimethoxyamphetamine (TMA) were detected in $6.25 \%$ of the samples as undeclared active pharmaceutical adulterants (Table 1), (Figures 2 and 3)

Furthermore, a combination of TMA and caffeine were detected in one distilled water sample; TMA, caffeine, and vitamin $\mathrm{E}$ combination was detected in one powder sample (Table 1).

\section{Discussion}

The present study results demonstrated that herbal weight loss products in Bojnurd (Northeast of Iran) were adulterated with caffeine, vitamin E, and TMA (an amphetamine analog). All the adulterated herbal products in this study were handmade and without Iran Food and Drug Organization (IFDO) license and approval. In addition, other medicines from pharmacological categories, such as thyroid hormones, rimonabant, laxatives, diuretics, antidepressants, and (Phosphodiesterase type 5) PDE5 inhibitors and opioids were not detected in the studied samples.

However, the adulteration of herbal medicines products, like slimming formulations, is a common global concern; pharmaceutical adulterants are frequently found in herbal weight loss products [12-19]. The World Health Organization (WHO) has definitions for substandard, spurious, falsely labeled, falsified, and counterfeit medicinal products.

Counterfeit medicine is deliberately and fraudulently mislabeled concerning identity or source. The counterfeit products may include those with the ingredients mentioned correctly or incorrectly, without active ingredients, with insufficient active ingredients, or with fake packaging. Besides, a counterfeit drug is a pharmaceutical product, i.e., produced and sold with the intent to deceptively representing its origin, effectiveness or authenticity, and may contain components, i.e., not declared on the label (which may or may not be harm- 
Table 1. Brand names, organoleptic characteristics, and active pharmaceutical ingredients detected in the adulterated herbal weight-loss

\begin{tabular}{|c|c|c|}
\hline Product Brand Name & Dose Form & $\begin{array}{l}\text { Detected Undeclared Pharmaceuti- } \\
\text { cal Ingredients }\end{array}$ \\
\hline Slim max & White hard capsules without imprint code & ND \\
\hline Laghari-e-si gol-e- Bustan & Green/transparent capsules without imprinted code & ND \\
\hline Green Fit & Silver hard capsule without imprint code & ND \\
\hline Lemon slim & Transparent soft gelatin capsule without imprint code & ND \\
\hline Cumin slim & Green soft gelatin capsules without imprint code & ND \\
\hline Mango slim & Beige capsules without imprinted code & ND \\
\hline Garcinia cambogia & Red/black capsules (imprint code: STP pharma) & ND \\
\hline Ronaless & Brown hard capsules without imprint code & ND \\
\hline Rogrin & Brown hard capsules without imprint code & ND \\
\hline Super Plant & Pink capsule without imprint code & ND \\
\hline Slim Full & Brown capsule without imprint code & ND \\
\hline Slimtex & Brown capsule without imprint code & ND \\
\hline Slim Quick & Olive- green tablet without imprint code & ND \\
\hline Cravil & Olive- green tablet without imprint code & Caffeine \\
\hline Garcinia cambogia & Beige tablet without imprint code & Caffeine \\
\hline Mohazel & Multi color tablet without imprint code & ND \\
\hline Caraway distilled water & $\begin{array}{l}\text { One-liter transplant plastic bottle containing colorless } \\
\text { transparent liquid with } \mathrm{pH}=9\end{array}$ & Caffeine \\
\hline Green cumin distilled water & $\begin{array}{l}\text { One-liter transplant plastic bottle containing hand- } \\
\text { made colorless transparent liquid with } \mathrm{pH}=9\end{array}$ & TMA/Caffeine \\
\hline Dill distilled water & $\begin{array}{l}\text { One-liter transplant plastic bottle containing hand- } \\
\text { made colorless transparent liquid with } \mathrm{pH}=8.5\end{array}$ & ND \\
\hline Celery distilled water & $\begin{array}{l}\text { One-liter transplant plastic bottle containing hand- } \\
\text { made colorless transparent liquid with } \mathrm{pH}=9\end{array}$ & Caffeine \\
\hline Apple vinegar & $\begin{array}{l}\text { One-liter transplant plastic bottle containing yellow } \\
\text { transparent liquid with } \mathrm{pH}=3\end{array}$ & ND \\
\hline Panj Giah & Hand-made olive color bulk powder & Caffeine/vitamin E \\
\hline Majoon-e- Lagari & Hand-made olive/brown color bulk powder & TMA/Caffeine/ vitamin E \\
\hline Dr. Sina slimming tea & A 3 -gram sachet containing green powder & ND \\
\hline Brunel slimming herbal tea & A 3 -gram sachet containing green powder & ND \\
\hline Aloe Vera super gel & A blue topical gel & ND \\
\hline Lemon anti-obesity drop & A transparent liquid with $\mathrm{pH}=5$ & ND \\
\hline Cumin anti-obesity drop & A transparent liquid with $\mathrm{pH}=8$ & ND \\
\hline
\end{tabular}

ND: Not Detected

Herbal medicine products obtained from pharmacies and herb shops in Bojnurd City, Iran. 

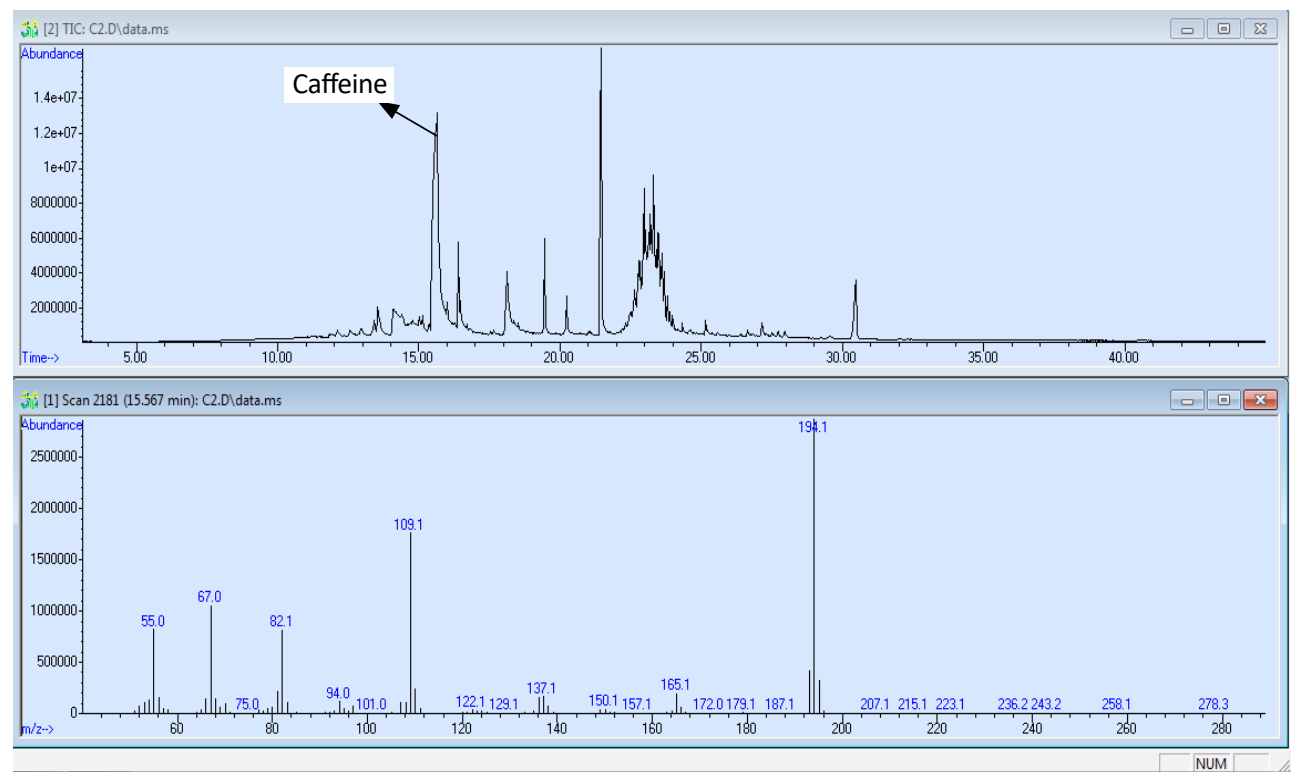

International Journal of
Medical Toxicology \& Forensic Medicin

Figure 1. The GC-MS chromatogram and mass spectrogram of the herbal medicine sample adulterated with caffeine

ful), or maybe supplied with inaccurate or fake packaging and labeling [20].

Caffeine was the most common adulterants in our study. Caffeine was detected in $>40 \%$ of the study samples; however, the presence of this substance is due to the use of an herbal source of this compound in the products' formulation. For example, green coffee and green tea (as main natural sources of caffeine) are the main used herbal components in the weight loss herbal products. Accordingly, only products have been considered as adulterated, which caffeine was not declared in the label as an ingredient. This finding is concordance with previous studies.
For example, Dastjerdi et al. argued that caffeine is the main adulterant in herbal weight loss supplements sold in Kermanshah City (northwest Iran) [19]. In another study in Kermanshah City, Iran, caffeine with phenolphthalein, phendimetrazine, and protriptyline were reported as common adulterants in alleged herbal slimming products [12]. Cianchino et al. detected caffeine, norephedrine, and furosemide in 4 phytopharmaceutical dosage forms for use in weight control programs, applying a validated capillary electrophoresis analytical method [14].

Caffeine, as methyl-xanthine derivatives and adrenergic stimulants, is frequently added to food and herbal

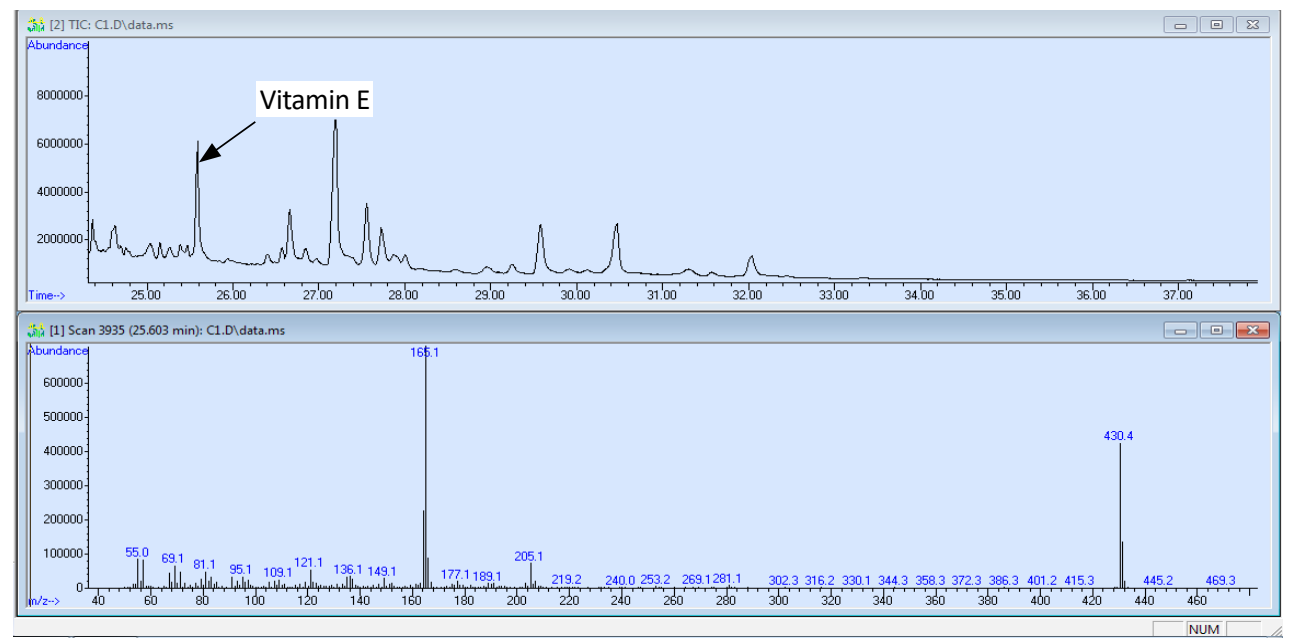

International J

Medical Toxicology \& forensic Medicine

Figure 2. The GC-MS chromatogram and mass spectrogram of the herbal medicine sample adulterated with vitamin E 


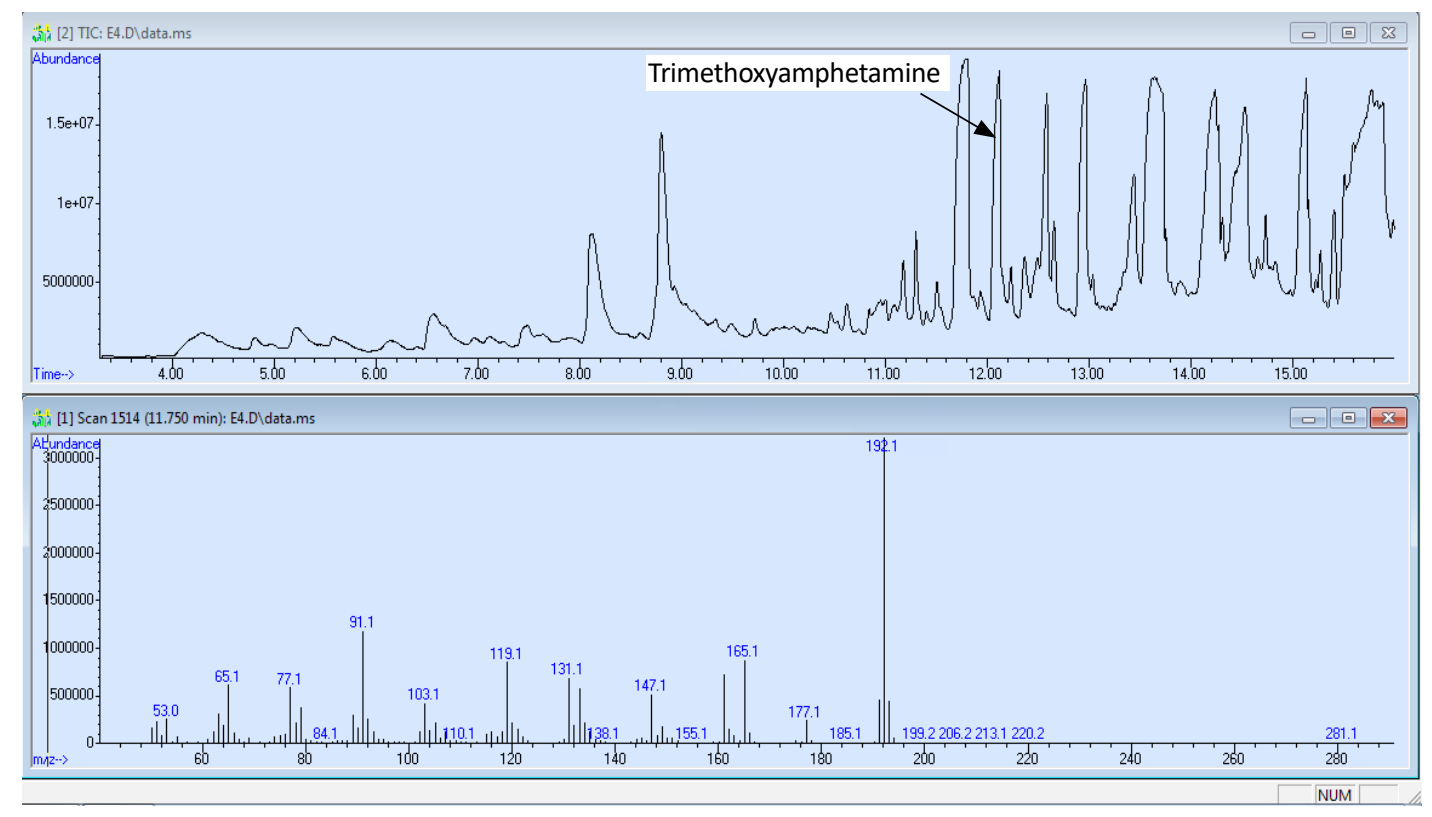

International Journal of
Medical Toxicology \&

Figure 3. GC-MS chromatogram and mass spectrogram of the herbal medicine sample adulterated with trimethoxyamphetamine

supplements due to their stimulating and thermogenic effects [21]. A meta-analysis investigation indicated that caffeine intake (congaing in a weight loss supplement) might promote bodyweight reduction [22]. However, there are reports of the adverse outcomes associated with caffeine uses in non-prescription herbal and nutritional supplements. Therefore, restricting the use of these products is currently being considered. Caffeine is unsafe when consumed orally for a long time or in high doses. Caffeine could cause insomnia, nervousness and restlessness, stomach irritation, nausea and vomiting, increased heart rate, and respiration.

Caffeine use could generate sleep disorders, and headache, anxiety, agitation, and chest pain in high doses [23]. Pendleton et al. reported a case of possible caffeineinduced seizure in a 38-year-old female taking an overthe-counter weight loss supplement containing caffeine (Zantrex - $3^{\mathrm{TM}}$ ). She experienced blurred vision and a new onset grand mal seizure with a two-month history of taking the supplement, i.e., advertised as a weightloss supplement [24].

One of the main and novel results of the present study was detecting TMA as an adulterant of the handmade herbal weight loss samples. To our knowledge, this is the first report about the presence of this substance, as an adulterant, in herbal slimming products. TMAs are a family of isomeric hallucinogenic amphetamines. There exist 6 different TMAs that only differ in the position of the 3 methoxy groups. Additionally, the
TMAs are analogs of the phenethylamine -derived alkaloid mescaline. These isomers are significantly as active as hallucinogenic drugs and have been placed into illegal drug schedules in some countries.

TMAs use develops various psychological conditions, ranging from sadness to empathy and euphoria. [25]. The loss of appetite is a common side effect of amphetamine stimulants. Accordingly, pharmaceutical amphetamine analogs, such as amfepramone, fenfluramine, sibutramine, fenproporex which inhibit serotonergic and noradrenergic reuptake, seem to be the most common adulterant in herbal weight loss products $[1,13,26]$. Moreover, consuming these adultered herbal weight loss products may be associated with psychiatric disorders due to their intrinsic toxicity [27].

Vitamin E was detected as another active pharmaceutical in the herbal samples in the present study. Vitamin E may have therapeutic effects on herbal products' consumers; however, due to the lack of its declaration in the explored products' label, it was considered as adulteration. Vitamin E is a lipid-soluble antioxidant, and its deficiency is associated with anemia, as well as neurological and several health disorders [28]. Besides, vitamin E may be a promising agent for attenuating metabolic syndrome, a medical condition inclusive of central obesity, hyperglycemia, hypertension, and dyslipidemia. Vitamin E has anti-oxidative, anti-inflammatory, antihypercholesterolemic, anti-obesity, anti-hypertensive, and anti-hyperglycemic properties. The pathways regu- 
lated by vitamin $\mathrm{E}$ are critical in developing metabolic syndrome, and it may exert some therapeutic benefits on this syndrome [29]. However, the efficacy and direct effects of vitamin $\mathrm{E}$ on weight loss remain unclear.

A study limitation was the qualitative analysis of the products; therefore, the quantification of the analysis are suggested for future studies.

\section{Conclusion}

In the study, caffeine, TMA, and vitamin E were identified as the synthetic adulterants in the handmade herbal slimming products. All substances identified in the study samples were not declared on the products' labels. Caffeine was the most common adulterant used in herbal slimming products. TMA, as an anti-appetite substance, has hallucinogenic effects. The current study reported the presence of TMA as adulterants in herbal weight loss products for the first time. Finally, due to the public interest in the extensive uses of herbal weight loss products, it is necessary to create awareness through health authorities.

\section{Ethical Considerations}

\section{Compliance with ethical guidelines}

This study was approved in the Ethics Committee of Research Deputy of the North Khorasan University of Medical sciences (Grant No. 1396-961).

\section{Funding}

This study has been financially supported by the of Research Deputy of the North Khorasan University of Medical sciences (Grant No. 1396-961).

\section{Author's contributions}

All authors contributed in preparing this article.

\section{Conflict of interest}

The authors declared no conflicts of interest.

\section{Acknowledgements}

The authors would like to thank the North Khorasan University of Medical Sciences, Bojnurd- Iran for providing financial support to conduct this research. The authors also wish to thank all technical staff of the forensic toxicology laboratory of legal medicine center, Bojnurd-Iran for their invaluable assistance

\section{References}

[1] Skalicka-Woźniak K, Georgiev MI, Orhan IE. Adulteration of herbal sexual enhancers and slimmers: The wish for better sexual well-being and perfect body can be risky. Food and Chemical Toxicology. 2017; 108:355-64. [DOI:10.1016/j. fct.2016.06.018] [PMID]

[2] Ekor M. The growing use of herbal medicines: Issues relating to adverse reactions and challenges in monitoring safety. Frontiers in Pharmacology. 2014; 4:177. [DOI:10.3389/ fphar.2013.00177] [PMID] [PMCID]

[3] Farrington R, Musgrave IF, Byard RW. Evidence for the efficacy and safety of herbal weight loss preparations. Journal of Integrative Medicine. 2019;17(2):87-92. [DOI:10.1016/j. joim.2019.01.009] [PMID]

[4] Poswal FS, Russell G, Mackonochie M, MacLennan E, Adukwu EC, Rolfe V. Herbal Teas and their health benefits: A scoping review. Plant Foods for Human Nutrition. 2019; 74(3):26676. [DOI:10.1007/s11130-019-00750-w] [PMID]

[5] Mateescu C, Popescu AM, Radu GL, Onisei T, Raducanu AE. Spectroscopic and spectrometric methods used for the screening of certain herbal food supplements suspected of adulteration. Advanced Pharmceutical Bulletin. 2017; 7(2):251-9. [DOI:10.15171/apb.2017.030] [PMID] [PMCID]

[6] Posadzki P, Watson L, Ernst E. Contamination and adulteration of Herbal Medicinal Products (HMPs): An overview of systematic reviews. European Journal of Clinical Pharmacology. 2013; 69(3):295-307. [DOI:10.1007/s00228-012-1353-z] [PMID]

[7] Kalman DS. An acute clinical trial evaluating the cardiovascular effects of an herbal ephedra-caffeine weight loss product in healthy overweight adults. International Journal of Obesity and Related Metaboilc Disorders. 2004; 28(10):1355-6. [DOI:10.1038/sj.ijo.0802418] [PMID]

[8] Zheng EX, Navarro VJ. Liver Injury from herbal, dietary, and weight loss supplements: A review. Journal of Clinical Translational Hepatology. 2015; 3(2):93-8. [DOI:10.14218/ JCTH.2015.00006] [PMID] [PMCID]

[9] Lee YX, Kwan YH, Lim KK, Tan CS, Lui NL, Phang JK, et al. A systematic review of the association of obesity with the outcomes of inflammatory rheumatic diseases. Singapore Medical Journal. 2019; 60(6):270-80. [DOI:10.11622/smedj.2019057] [PMID] [PMCID]

[10] Carbone S Canada JM, Billingsley HE, Siddiqui MS, Elagizi A, Lavie CJ. Obesity paradox in cardiovascular disease: Where do we stand? Vascular and Health Risk Management. 2019; 15:89-100. [DOI:10.2147/VHRM.S168946] [PMID] [PMCID]

[11] Avgerinos KI, Spyrou N, Mantzoros CS, Dalamaga M. Obesity and cancer risk: Emerging biological mechanisms and perspectives. Metabolism. 2019; 92:121-35. [DOI:10.1016/j. metabol.2018.11.001] [PMID]

[12] Fakhri S, Mohammadi B, Jalili R, Hajialyani M, Bahrami $\mathrm{G}$. Screening and confirmation of different synthetic adulterants in slimming products. Asian Journal of Pharmaceutical and Clinical Research. 2018; 11(2):260-4. [DOI:10.22159/ ajpcr.2018.v11i2.22516]

[13] De Carvalho LM, Cohen PA, Silva CV, Moreira AP, Falcão TM, Dal Molin TR, et al. A new approach to determining 
pharmacologic adulteration of herbal weight loss products. Food Additives \& Contaminants: Part A: Chemistry, Analysis, Control, Exposure \& Risk Assessment. 2012; 29(11):1661-7. [DOI:10.1080/19440049.2012.706834] [PMID]

[14] Cianchino V, Acosta G, Ortega C, Martínez LD, Gomez MR. Analysis of potential adulteration in herbal medicines and dietary supplements for the weight control by capillary electrophoresis. Food Chemistry. 2008; 108(3):1075-81. [DOI:10.1016/j.foodchem.2007.11.042] [PMID]

[15] Pamukcu Gunaydin G, Dogan NO, Levent S, Kurtoglu Celik G. Herbal weight loss pill overdose: Sibutramine hidden in pepper pill. Case Reports in Emergency Medicine. 2015; 2015:213874. [DOI:10.1155/2015/213874] [PMID] [PMCID]

[16] Khazan M, Hedayati M, Kobarfard F, Askari S, Azizi F. Identification and determination of synthetic pharmaceuticals as adulterants in eight common herbal weight loss supplements. Iran Red Crescent Medical Journal. 2014; 16(3):e15344. [DOI:10.5812/ircmj.15344] [PMID] [PMCID]

[17] Moreira AP, Motta MJ, Dal Molin TR, Viana C, de Carvalho LM. Determination of diuretics and laxatives as adulterants in herbal formulations for weight loss. Food Additives \& Contaminants: Part A: Chemistry, Analysis, Control, Exposure \& Risk Assessment. 2013; 30(7):1230-7. [DOI:10.1080/19440049. 2013.800649] [PMID]

[18] Reeuwijk NM, Venhuis BJ, de Kaste D, Hoogenboom RL, Rietjens IM, Martena MJ. Active pharmaceutical ingredients detected in herbal food supplements for weight loss sampled on the Dutch market. Food Additives \& Contaminants: Part A: Chemistry, Analysis, Control, Exposure \& Risk Assessment. 2014; 31(11):1783-93. [DOI:10.1080/19440049.2014.958 574] [PMID]

[19] Dastjerdi AG, Akhgari M, Kamali A, Mousavi Z. Principal component analysis of synthetic adulterants in herbal supplements advertised as weight loss drugs. Complementary Therapies in Clinical Practice. 2018; 31:236-41. [DOI:10.1016/j. ctcp.2018.03.007] [PMID]

[20] World Health Organization. Counterfeit drugs: Guidelines for the development of measures to combat counterfeit drugs [Internet]. 2019 [Updated 2019 July 2]. Available from: http://apps.who.int/medicinedocs/en/d/Jh1456e/2.1.html

[21] Viana C, Zemolin GM, Müller LS, Dal Molin TR, Seiffert H, de Carvalho LM. Liquid chromatographic determination of caffeine and adrenergic stimulants in food supplements sold in Brazilian e-commerce for weight loss and physical fitness. Food Additives \& Contaminants: Part A: Chemistry, Analysis, Control, Exposure \& Risk Assessment. 2016; 33(1):1-9. [D OI:10.1080/19440049.2015.1112040] [PMID]

[22] Tabrizi R, Saneei P, Lankarani KB, Akbari M, Kolahdooz F, Esmaillzadeh A, et al. The effects of caffeine intake on weight loss: A systematic review and dos-response meta-analysis of randomized controlled trials. Critical Reviews in Food Science and Nutrition. 2019; 59(16):2688-96. [DOI:10.1080/1040 8398.2018.1507996] [PMID]

[23] WebMed. Caffeine [Internet]. 2019 [Updated 2019 Jan 30] Available from: https://www.webmd.com/vitamins/ai/ingredientmono-979/caffeine

[24] Pendleton M, Brown S, Thomas CM, Odle B. Potential toxicity of caffeine when used as a dietary supplement for weight loss. Journal of Dietary Supplements. 2013; 10(1):1-5. [DOI:10. 3109/19390211.2012.758215] [PMID]
[25] Nieddu M, Boatto G, Pirisi MA, Azara E, Marchetti M LC-MS analysis of trimethoxyamphetamine designer drugs (TMA series) from urine samples. Jornal of Chromatography B. 2008; 867(1):126-30. [DOI:10.1016/j.jchromb.2008.03.027] [PMID]

[26] Bryant SM, Lozada C, Wahl M. A Chinese herbal weight loss product adulterated with fenfluramine. Annals of Emergency Medicine. 2005; 46(2):208. [DOI:10.1016/j.annemergmed.2005.03.021] [PMID]

[27] Bersani FS, Coviello M, Imperatori C, Francesconi M, Hough CM, Valeriani G, et al. Adverse psychiatric effects associated with herbal weight-loss products. Biomedcal Research Intrnational. 2015; 2015:120679. [DOI:10.1155/2015/120679] [PMID] [PMCID]

[28] Malik A, Eggersdorfer M, Trilok-Kumar G. Vitamin E status in healthy population in Asia: A review of current literature. International Journal for Vitamin and Nutrition Research. 2019:1-14. [DOI:10.1024/0300-9831/a000590] [PMID]

[29] Wong SK, Chin KY, Suhaimi FH, Ahmad F, Ima-Nirwana S. Vitamin E as a potential interventional treatment for metabolic syndrome: Vidence from animal and human studies. Frontiers in Pharmacology. 2017; 8:444. [DOI:10.3389/ fphar.2017.00444] [PMID] [PMCID] 